\title{
Detector cintilador semicondutor para radiação gama
}

\author{
F. T. V. der Laan ${ }^{\text {a }}$; V. Borges ${ }^{\text {a }}$; J. R. S. Zabadal ${ }^{\text {a }}$ \\ ${ }^{a}$ Grupo de Estudos Nucleares do Departamento de Engenharia Mecânica (GENUC/DEMEC) \\ Universidade Federal do Rio Grande do Sul (UFRGS) \\ Av. Osvaldo Aranha, $n^{\circ}$ 99, $4^{\circ}$ andar \\ 90035-190 Porto Alegre, RS \\ ftvdl@ufrgs.br \\ borges@ufrgs.br \\ jorge.zabadal@ufrgs.br
}

\begin{abstract}
Nowadays the devices employed to evaluate individual radiation exposition are based on dosimetric films and termoluminescent crystals, whose measurements must be processed in specific transductors. Hence, these devices carry out indirect measurements. Although a new generation of detectors based on semiconductors which are employed in EPD's (Eletronic Personal Dosimeters) being yet available, it high producing costs and large dimensions prevents the application in personal dosimetry. Recent research works reports the development of new detection devices based on photovoltaic PIN diodes, which were succesfully employed for detecting and monitoring exposition to X rays. In this work, we step forward by coupling a $2 \mathrm{~mm}$ antracene scintillator NE1, which converts the high energy radiation in visible light, generating a Strong signal which allows dispensing the use of photomultipliers. A low gain high performance amplifier and a digital acquisition device are employed to measure instantaneous and cummulative dosis for energies ranging from $\mathrm{X}$ rays to Gamma radiation up to $2 \mathrm{MeV}$. One of the most important features of the PIN diode relies in the fact that it can be employed as a detector for ionization radiation, since it requires a small energy amount for releasing electrons. Since the photodiode does not amplify the corresponding photon current, it must be coupled to a low gain amplifier. Therefore, the new sensor works as a scintillator coupled with a photodiode PIN. Preliminary experiments are being performed with this sensor, showing good results for a wide range of energy spectrum.
\end{abstract}

Keywords: Nuclear Instrumentation Detectors X and Gama, photovoltaic sensor, personal dosimetry. 


\section{INTRODUCÃO}

Detectores de radiações são amplamente empregados em todos os setores que fazem uso das radiações ionizantes. Existem diversos processos pelos quais diferentes tipos de radiações podem interagir com o meio sensível do detector para medir ou indicar características dessas radiações. Atualmente diversos tipos e modelos de detectores encontram-se disponíveis no mercado, a escolha conveniente desse equipamento dependendo de fatores técnicos e dos custos envolvidos e, principalmente, do uso a que se destina. Detectores que apresentam alta eficiência na detecção X e gama são dispositivos, cujo meio sensível são formados por materiais cintiladores ou semicondutores, que são amplamente empregados nas diversas áreas de pesquisas e monitoração, podendo também ser utilizados na espectrometria de raios-X e gama in situ [1].

Os cristais inorgânicos, como o germânio e o silício, são objetos de estudados desde os anos 50 para aplicação como sensores de radiação ionizantes. Devido a sua eficiência e ao crescente uso, houve uma necessidade de produção em larga escala desses cristais e outros compostos. Pesquisas recentes estão sendo desenvolvidas para obtenção desses cristais, atendendo a critérios de simplicidade e custo compatível com outros detectores empregados em radiações ionizantes [2].

Os cintiladores são formados por materiais sólidos, líquidos e gasosos, que produzem fluorescência quando a radiação interage com os mesmos [3]. A conversão de luz, transformada em corrente elétrica, é feita pela utilização de fotomultiplicadoras, que são dispositivos acoplados opticamente ao elemento cintilador. Os detectores cintiladores, que para operarem, necessitam de criogenia, como os de Ge-Li, fazem uso de nitrogênio líquido, e de um amplo sistema de detecção. O uso do antraceno como detector de radiação teve seus estudos iniciados na década de 50 e pesquisas recentes mostram, que esse cintilador orgânico apresenta linearidade com a dose absorvida, para fótons com megavoltagens $(1,25 \mathrm{MeV})$, podendo ser aplicado na medida de dose absorvida em radioterapia [7].

Os detectores semicondutores, também denominados de detectores de estado sólido, são constituídos de substâncias cristalinas puras, ou dopadas com determinadas impurezas. Nestes detectores os elétrons situam-se em bandas de energia bem definidas e separadas pela chamada banda proibida. Estes tipos de detectores trabalham à temperatura ambiente, não necessitando de nenhum sistema de refrigeração.

Na monitoração individual são utilizados, principalmente, dosímetros termoluminescentes, cujos sistemas necessitam de filtros adequados, que dependem da energia da radiação que se está monitorando. Estes dosímetros são bastante práticos e amplamente utilizados na dosimetria pessoal, mas são dispositivos de leitura indireta, cujo sinal associado à dose de radiação necessita ser processado.

O uso de fotodiodos na dosimetria das radiações ionizantes, tanto individual como de área, tem a vantagem de apresentar a resposta linear com a dose de radiação recebida. Além disso, o fotodi- 
odo produz uma corrente elétrica aproximadamente 18.000 vezes maior do que a produzida em uma câmara de ionização de mesmo volume. Portanto é uma alternativa bastante promissora de economia de espaço, potência elétrica e redução de peso do equipamento.

Tendo em vista a necessidade de um detector de radiação que tenha alta eficiência na detecção de raios $\mathrm{X}$ e gama de baixa energia e que possa ser facilmente utilizado na monitoração de área e individual, devido ao seu baixo custo e dimensões e que opera na temperatura ambiente, foi desenvolvido o projeto de um sensor que utiliza um diodo fotovoltaico [4]. Este sensor de radiação gama semicondutor é constituído de um diodo fotovoltaico encapsulado, com o propósito de bloquear a luz, para que apenas a radiação que atravessa a cápsula interaja com a fotocélula. Com um amplificador de ganho na ordem de $10^{8}$ retira-se um nível de sinal na ordem de mVolts, com ruído na ordem $\mu$ Volts. Os ensaios para verificar o funcionamento desse detector foram feitos somente para raios $\mathrm{X}$, com energia de até $100 \mathrm{keV}$ e corrente de tubo de até $100 \mathrm{~mA}$. Os resultados dos testes mostraram que o sistema desenvolvido é adequado para a detecção e a monitoração da exposição a raios-X. O sistema de detecção apresentou viabilidade para a construção de sensores eletrônicos portáteis, cuja principal vantagem é o emprego como dosímetros com leitura direta, em tempo real, podendo vir a ser um substituto adequado para as canetas dosimétricas, porém, mecanicamente, mais resistente e a um custo menor [5].

Um novo sistema de detecção está sendo testado para radiação X e gama de até $2 \mathrm{MeV}$. Este novo sistema pode reunir as vantagens de fotodiodos e de materiais cintiladores. O sistema consiste basicamente de um cristal cintilador de antraceno, de camada fina, acoplado na superfície de um fotodiodo. Testes estão sendo realizados para verificar se a introdução dessa fina camada de material intensificador na frente do diodo poderá eliminar a necessidade do uso de um circuito amplificador ou utilizar amplificadores de baixo ganho, o que poderá acarretar redução nos custos do sistema, mantendo a mesma eficiência.

\section{MATERIAIS E MÉTODOS}

Estudos anteriores foram realizados com um fotodiodo tipo PIN, que consiste de uma junção PN com camada intrínsica, acoplado a um amplificador. Os testes mostraram a viabilidade desse sistema para radiação $\mathrm{X}$ de até $100 \mathrm{keV}$ [5].

O sistema estudado consiste de um diodo fotovoltaico inserido em uma cápsula fechada que não permite a passagem de luz visível. O sinal resultante da interação da radiação ionizante (raios X ou gama) foi amplificado através de um amplificador operacional tipo OPA128 e LF357. O amplificador LF357 foi incialmente testado, sendo posteriormente substituído pelo OPA128, que apresentou melhor confiabilidade, pois apesar de todos os ajustes recomendados, não foi obtido o padrão zero de saída do amplificador LF357. O amplificador OPA128 apresenta características operacionais superiores e conseguiu-se manter o sinal de saída estável com baixo nível de ruído. Todo o sistema do circuito amplificador foi alimentado com duas baterias alcalinas de 9,0 Volts. 
Os testes para esse sistema foram realizados com um aparelho de raios $\mathrm{X}$, com tubo de ânodo fixo, feixe colimado, tamanho de campo fixo e constante e filtração inerente constante, sem uso de filtração adicional. O tempo de exposição foi definido para 1,0 s e a distância do tubo ao elemento sensor permaneceu fixa em $0,1 \mathrm{~m}$. Toda a geometria do aparato experimental foi mantida inalterada durante os testes.

Foram realizados quatro conjuntos de testes, com as seguintes tensões e correntes de tubo do aparelho de raios X: 1- tensões de tubo $40 \mathrm{keV}, 45 \mathrm{keV}$ e $50 \mathrm{keV}$ com corrente de tubo constante de $100 \mathrm{~mA}$; 2- tensões de tubo de $55 \mathrm{keV}, 60 \mathrm{keV}$ e $65 \mathrm{keV}$ com corrente de tubo constante de $80 \mathrm{~mA}$; 3- tensões de tubo de $70 \mathrm{keV}, 75 \mathrm{keV}$ e $80 \mathrm{keV}$ com corrente de tubo constante de 60 $\mathrm{mA}$; 4- tensões de tubo $85 \mathrm{keV}, 90 \mathrm{keV}$ e $100 \mathrm{keV}$ com corrente de tubo constante de $40 \mathrm{~mA}$.

Todos estes conjuntos de testes mostraram uma relação crescente do sinal de saída do sensor com o aumento da tensão (energia) de tubo do aparelho de raios X, para correntes de tubo constantes [5].

Foi estudada também, separadamente, a sensibilidade da resposta do sensor em função somente da tensão (energia) do feixe de radiação e em função somente da corrente de tubo. Esse estudo foi realizado normalizando a tensão de saída do sistema sensor, através da equação que relaciona a intensidade relativa do feixe de raios X com a alta-tensão aplicada no tubo, com a intensidade de corrente de tubo, com o tempo de exposição e a distância do tubo ao sensor [6].

Os resultados das tensões normalizadas de saída do sistema mostraram que, à medida que aumenta a energia do feixe de raios $\mathrm{X}$ aumenta o sinal de saída do sistema detector e, à medida que aumenta a corrente de tubo de raios X, também aumenta o sinal de saída do sistema, conforme mostrado nas Figuras 1 e 2. Esses resultados indicam a viabilidade do uso desse sistema na detecção de radiação $X$.

\section{RESULTADOS E DISCUSSÕES}

Verificada a viabilidade do uso do sistema PIN para raios X, outros estudos foram desenvolvidos para o emprego na detecção da radiação gama. Inicialmente o mesmo sistema anterior foi testado para medidas com fontes de Cs-137 e Co-60. Foi montado um aparato experimental com as fontes colimadas e mantidas a $0,5 \mathrm{~m}$ do sistema detector. A fonte de Cs-137 utilizada possuía atividade de 3,7 GBq e a de Co-60 com 0,13 GBq.

Os resultados das tensões de saída do sistema mostraram que, à medida que aumenta as exposições ou doses, aumenta o sinal de saída do sistema de detecção. Verificou-se também que, com o aumento da energia da radiação gama aumenta também o sinal de saída do sistema detector, mostrando uma fraca dependência energética do sistema. Estudos estão sendo realizados para determinar essa dependência energética e incluem também variações na atividade das fontes, nas 
distâncias, nas exposições ou doses e nas taxas de exposições. Estes estudos incluem a normalização das tensões de saída do sistema em função desses parâmetros.

$\mathrm{Na}$ continuidade desses estudos foi desenvolvido um novo sensor, acoplando um cristal cintilador de antraceno de $2 \mathrm{~mm}$ tipo NE1, capaz de converter a radiação X e/ou gama para a banda de energia da luz visível. As medidas para esse novo sistema foram realizadas com o mesmo aparato experimental anterior, descrito anteriormente, tanto para detectar raios $\mathrm{X}$ como radiação gama. Os testes de detecção para raios X mostraram que as tensões normalizadas de saída do sistema aumentam com o aumento da energia do tubo, para correntes de tubo constantes, pois aumenta a intensidade do feixe de radiação, conforme mostrado na Figura 1. Esses testes também indicaram que o sinal normalizado de saída do sistema aumenta com o aumento da corrente de tubo, para tensões constantes, como mostra a Figura 2.

Figura 1: Tensão normalizada de saída do sensor em função da alta tensão do tubo de raios X.

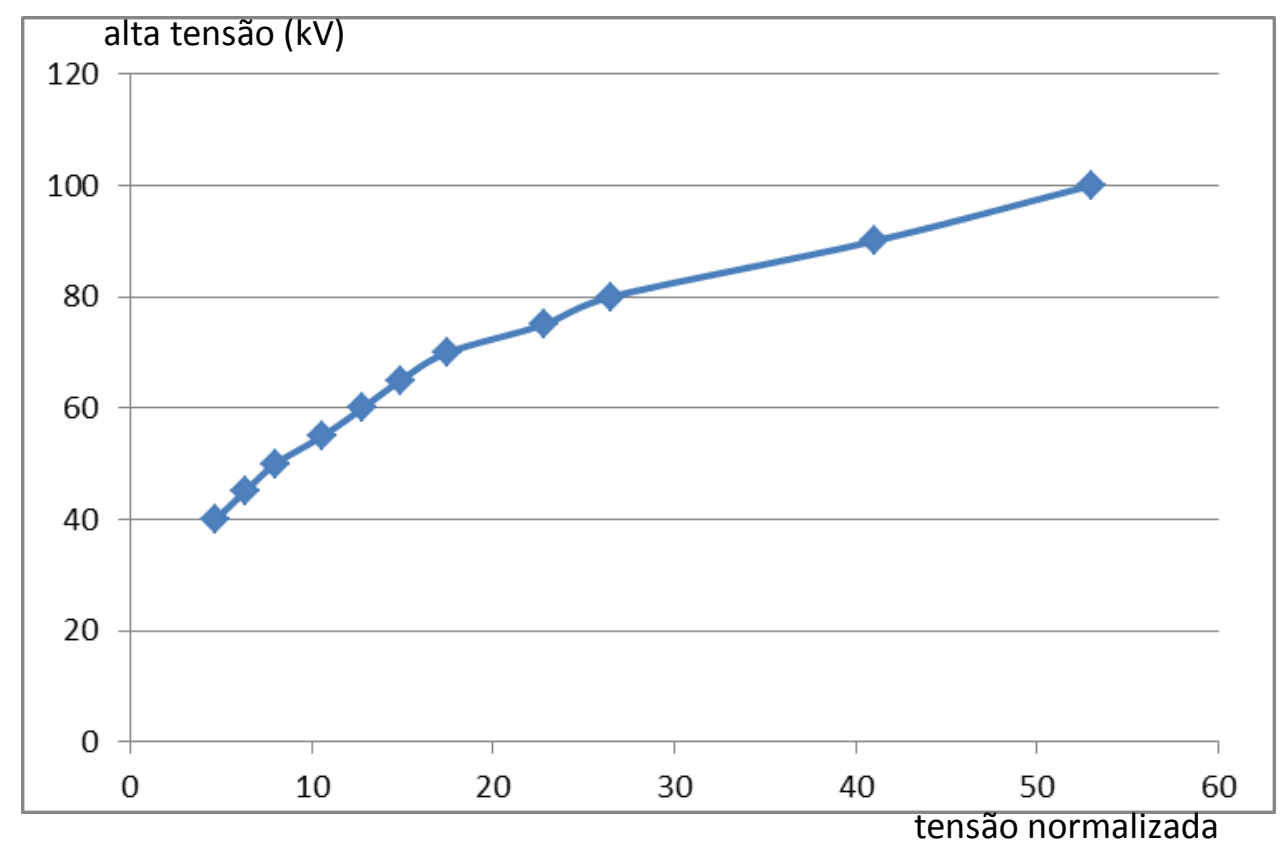

Fonte: Autores

Esse novo sistema foi testado para detecção de radiação gama. Nos testes experimentais foi utilizado um amplificador de alto desempenho, mas de baixo ganho e um sistema de leitura digital. Os resultados das tensões de saída do sistema mostraram as mesmas características qualitativa 
anteriores, ou seja, à medida que aumenta a dose de radiação gama aumenta também o sinal de saída do sistema detector, e que o mesmo mostra uma fraca dependência energética do sistema.

Figura 2: Tensão normalizada de saída do sensor em função da corrente de tubo aplicada no equipamento de raios $\mathrm{X}$.

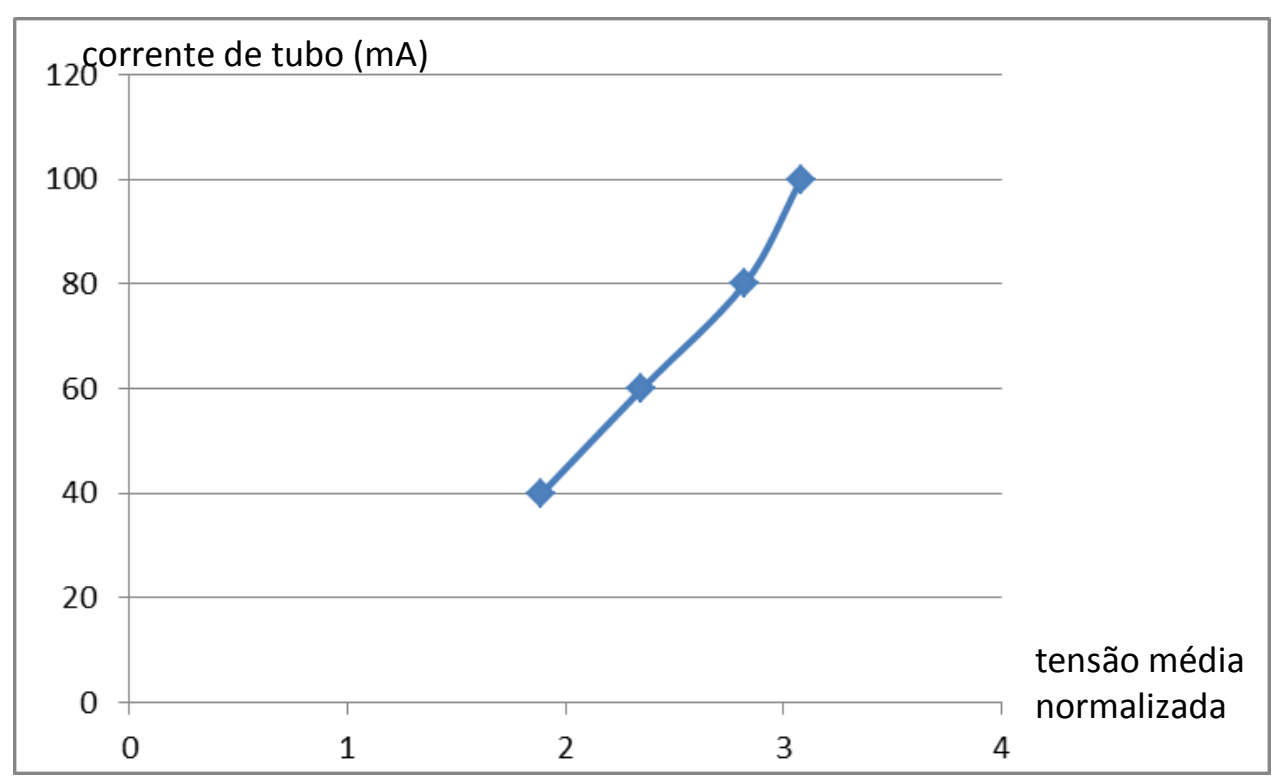

Fonte: Autores

Novos estudos estão sendo realizados para determinar essa dependência energética, bem como a sensibilidade do sinal de saída em função da dose e das taxas de dose. Além disso, os estudos incluem também a dependência da direção de incidência do feixe de radiação e variações na atividade das fontes e nas distâncias. Estes estudos incluem a normalização das tensões de saída do sistema em função desses parâmetros.

\section{CONCLUSÕES}

Esse novo sistema de detecção de radiação em estudo, reúne em um único sensor, as vantagens dos sistemas que fazem uso de fotodiodos e materiais cintiladores. Os testes realizados mostram que o sistema dispensa o uso de fotomultiplicadoras, indispensáveis nos sistemas unicamente 
cintiladores, e necessita somente de um amplificador comercial de baixo ganho, pois a conversão da radiação ionizante de alta energia para a banda visível, no cristal cintilador, produz um forte sinal, permitindo com isso dispensar o uso de fotomultiplicadoras. Os testes preliminares mostraram a viabilidade do uso desse sistema para a detecção de radiação X e gama, permitindo a construção de sensores eletrônicos portáteis de baixo custo, de leitura direta de dose e/ou exposição à radiação, podendo vir a ser empregado na dosimetria pessoal.

\section{REFERÊNCIAS}

1. J. S. Fernandes, C. R. Appoloni, A.C. Andrello, "Aplicabilidade de um sistema portátil de espectrometria de raios-X e raios gama in situ”, International Nuclear Atlantic Conference INAC 2005, Santos, SP, Brazil, August 28 to September 2. (2005).

2. V. C. Cortese, M. C. C. Pereira, M. M. Hamada, "Crescimento de cristais baseados em Iodeto de Césio (CsI) para aplicação como detectores de radiação”, International Nuclear Atlantic Conference - INAC 2005, Santos, SP, Brazil, August 28 to September 2. (2005).

3. N. Tsoulfanidis, "Measurement and Detection of Radiation", Taylor \& Francis Publisher, USA (1995).

4. F. T. van der Laan, "Medidor não invasivo de alta tensão, tempo e exposição em aparelhos de raios- $X$ ”. Dissertação de Mestrado. Universidade Federal do Rio Grande do Sul. Porto Alegre (1996).

5. F. T. van der Laan, V. Borges, J. R. S. Zabadal, "Detector para raios X de baixa energia, usando diodo PIN fotovoltaico", Revista Liberato, Volume 14, n. 21, jan/jun, pp. 91-98 (2013).

6. S. Bushong, "Radiologic Science for Technologist: physics, biology and protection”, Moaby Elsevier, Canadá (2008). 
7. R.A. Rubo, "Dosímetro de cintilação de antraceno para uso na dosimetria de magavoltagem”. Dissertação de Mestrado. Faculdade de Filosofia, Ciências e Letras da Universidade de São Paulo. São Paulo (2001). 